\section{Economic analysis in patient safety: a neglected necessity}

\section{David Meltzer}

Two articles in this issue ${ }^{12}$ appraise economic analyses related to patient safety. Among many findings, one stands out: there are not very many analyses to appraise. Several questions present themselves immediately: Should we care? If yes, why are there not more such studies? And, what can be done if we want fill this void?

\section{SHOULD WE CARE?}

It is not difficult to understand why passionate advocates for patient safety might argue we should not. The idea that healthcare should be allowed to cause injury when injury could have been avoided is a difficult one for many who have felt the pain of medical error. Moreover, the case that medical error is costly has been so heavily emphasised that many may view reduction of medical error as cost-saving. The articles by Etchells et $a l$ and de Rezende et al in this issue do identify some studies in which such cost savings are evident; the ability to identify-such win-win interventions is a best case scenario for economic evaluation. However, these cases are by far the exception. In fact, this is as it should be: the purpose of patient safety initiatives is to make patients safer. This is evident even in considering the 'business

Department of Economics, Section of Hospital Medicine, The University of Chicago, Harris School of Public Policy, Center for Health and the Social Sciences, Chicago, USA

Correspondence to Dr David Meltzer, Department of Economics, Section of Hospital Medicine, The University of Chicago, Harris School of Public Policy, Center for Health and the Social Sciences, 5841 S Maryland MC 2007, Chicago, IL 60637, USA; dom2@uchicago.edu case' for patient safety; it is not just reduced costs, or even reduced malpractice settlements, but the increased demand for care that a provider experiences when they can offer patients care that is safer. Indeed, whether one takes the perspective of a patient, provider or society, if we are not spending money on some patient safety initiatives that cost more than they save, we are not spending enough on patient safety.

The corollary of this observation is that it is possible to spend too much on patient safety even when the consequence is that some persons suffer harm that could have been avoided. This is the case because the resources devoted to safety could have been used for other purposes. This is what economists refer to as 'opportunity costs'. How many pap smears, immunisations, or other costeffective medical interventions could have been provided with the same funds? This was perhaps less of concern when patient safety was in its infancy. But, today, the typical hospital employs many individuals who devote themselves full-time to efforts to improve patient safety so the opportunity costs associated with spending on patient safety cannot be ignored. Moreover, proper accounting of the cost of workflow changes and infrastructure investments, such as computerised physician order entry, that are done in the name of patient safety, could easily far exceed the cost of the more easily measured elements of the cost of patient safety efforts. Efforts to improve patient safety typically require foregoing other initiatives that could improve health.
WHY ARE THERE NOT MORE STUDIES?

The sense that patient safety should be pursued independent of its costs may be one reason that there are not more economic evaluations of patient safety initiatives. This is reinforced by the fact that for many healthcare institutions, quality and safety initiatives are heavily driven by public or private payers or accreditation agencies that have little or no direct incentives to consider the costs of their requirements. This is an obvious risk of unfunded or minimally funded mandates. Providers will feel intense pressure to comply with these initiatives even when they do not believe they are likely to be useful. These requirements may also inhibit the production of local knowledge about safety. There is little incentive for providers to perform economic evaluations of interventions if they are required to perform them to continue in business. Likewise, there is little incentive for regulators or payers to perform economic evaluations if they know that providers will follow their direction regardless and will have to bear the costs for doing so.

Another challenge is that economic evaluations of patient safety interventions are not easy to do well. de Rezende et al focus particularly on this, highlighting the absence of good data for all but a few patient safety interventions, and the high degree of effort required and challenges of transparency that arise when approaches such as simulation modelling are used to try to address data limitations. The sort of rigorous assessment of the quality of the literature provided by Etchells et al further reinforces how difficult it is to perform a really high quality economic evaluation-less than half a dozen studies met their rigorous standards. One must add to this the reality that the effectiveness, cost, and hence cost-effectiveness, of patient safety initiatives are often 
highly dependent on the context in which they are implemented. To the extent this is true, economic evaluations may be of little value unless they can be effectively tailored to reflect variations among the institutions that might implement the patient safety interventions under consideration. Economic evaluations may also be of little value in informing policy unless they can address the perspectives of multiple stakeholders whose behaviour they are intended to influence.

An additional set of challenges is technical in nature. It is often very difficult to measure costs in hospitals and other healthcare organisations, since many resources are fixed costs that serve many activities. As a result, such costs are difficult to allocate to specific activities and may not change even when activities that require that resource increase or decrease. ${ }^{3}$ For example, a program to decrease the use of MRI scans might produce almost no savings unless it resulted in reductions in staffing or capital spending. However, most costaccounting practices do not account for this. Fixed costs may also be challenging to account for accurately in healthcare because fixed costs are often difficult to allocate to specific activities. Accounting for the cost of time presents similar challenges because time use is often difficult to measure or attribute to a specific task. This is especially important because most healthcare costs derive ultimately from the cost of time allocated to activities.

Finally, when it comes to economic evaluations of interventions, simply measuring the harms and other costs associated with adverse events themselves may at best provide bounds on the benefits of the intervention unless a strong experimental design is applied. Unfortunately, regulatory and patient safety concerns often prevent such rigorous evaluations from being performed and almost never support their costs.
WHAT CAN BE DONE IF WE WANT TO FILL THIS VOID?

Given the myriad barriers described above, increasing the extent to which economic evaluations of safety practices are performed will require multiple efforts. First, it is imperative that the patient safety community embrace the idea that the growing societal commitment of resources to safety demands that the allocation of those resources be informed by rational analyses of their benefits and costs, both to obtain the greatest benefits from available resources for patient safety and to identify when patient safety resources would be better allocated to other social purposes. Second, it is critical that those in the position to dictate, or at least influence, patient safety practice-for example, regulators and payerscommit themselves to systematic evaluation of the benefits and costs of their policies. This means investing resources to perform rigorous clinical and economic evaluations of safety interventions they choose to promote. Third, we should invest in improving the methods for the evaluation of patient safety initiatives, focusing on the questions that are likely to be most salient empirically, for example, how to allocate fixed costs and time to specific activities. Given the high likelihood that the effectiveness of patient safety initiatives varies greatly across clinical contexts, it is especially important that we learn how to use local data to perform evaluations at low cost. Such learning will require investments at the national and international level to improve and codify knowledge about the economic evaluation of patient safety at the frontier of the field, and at the level of individual institutions to allow best practices to be disseminated and tested in practice.

\section{CONCLUSION}

Some in patient safety may resist addressing concerns about costs because they believe this will present barriers to broad dissemination of potentially valuable patient safety interventions. Advocates for other forms of medical spending, whether on professional services, new technologies, or pharmaceuticals, may have similar sentiments about their own approaches to improving health. Patients, and especially those who have personal experiences concerning safety, may have similar feelings, and politicians and persons with vested economic interests could easily play on those fears to promote investments in safety in the absence of evidence of value. It could be argued that an adverse outcome due to a medical error should be considered worse than an adverse outcome for some other reason. However, even influential statements on the importance of patient safety, such as the Institute of Medicine's Crossing the Quality Chasm report, ${ }^{4}$ do not privilege patient safety in this way, listing it first among the six dimensions of quality (safety, effectiveness, patient-centeredness, timeliness, efficiency, and equity), but not placing it above them.

Those who wish to challenge the use of economic evaluation to inform priorities for patient safety may also point to the methodological challenges presented by economic evaluation. These are important to recognise. Nevertheless, it is difficult to imagine that rational use of the tools of economic analysis to supplement expert judgement in prioritising patient safety interventions would produce worse decisions than expert judgement alone, and there are good reasons to expect them to produce better decisions. Moreover, the increasing use of economic analysis of patient safety is likely to strengthen its scientific basis and effectiveness in maximising the health improvements achieved through spending on patient safety. From its current position as a neglected necessity, it is critical that 
economic evaluation of patient safety become an essential tool for the field.

Funding Financial support for this work was provided by a Midcareer Career Development Award from the National Institute of Aging (1 K24 AG031326-01, PI Meltzer and the Agency for Healthcare Quality and Research through the Hospital Medicine and Economics Center for Education and Research in Therapeutics (CERT) (U18 HS016967-01, Meltzer, PI).
Competing interests None.

Provenance and peer review Commissioned; internally peer reviewed.

BMJ Qual Saf 2012;21:443-445. doi:10.1136/bmjqs-2012-001109

\section{REFERENCES}

1. Etchells $\mathrm{E}, \mathrm{Koo} \mathrm{M}$, Daneman $\mathrm{N}$, et al. Comparative economic analyses of patient safety improvement strategies in acute care: a systematic review. BMJ Qual Saf 2012;21:448-56.

2. De Rezende B A, Or Z, Com-Ruelle L, et al Economic evaluation in patient safety: a literature review of methods. BMJ Qual Saf 2012;21:457-65.

3. Rauh SS, Wadsworth EB, Weeks WB, et al. Clinical Quality Improvement Fails to Deliver Bottom-Line Results. N Engl J Med 2011;365:e48.

4. Institute of Medicine. Crossing the Quality Chasm: A New Health System for the Twenty-First Century. Washington: National Academy Press, 2001.
Advancing Postgraduates. Enhancing Healthcare.

The Postgraduate Medical Journal is dedicated to advancing the understanding of postgraduate medical education and training

- Acquire the necessary skills to deliver the highest possible standards of patient care

- Develop suitable training programmes for your trainees

- Maintain high standards after training ends

Published on behalf of the fellowship for Postgraduate Medicine

FOR MORE DETAILS OR TO SUBSCRIBE, VISIT THE WEBSITE TODAY 